\title{
Designing technologies for presence-in-absence: illustrating the Cube and the Picture Frame
}

Received: 1 October 2005/ Accepted: 15 October 2005/Published online: 1 August 2006

(C) Springer-Verlag London Limited 2006

\begin{abstract}
Presence-in-absence is a subjective feeling of a significant other when he or she is not physically copresent and several emerging technologies increasingly aim to support this feeling of presence. However, no established framework for designing presence-in-absence technologies exists. We report on the design and evaluation of two novel technologies for supporting the experience of an absent significant other being present i.e. presence-in-absence. On the basis of a longitudinal field study of communication in romantic relationships, we constructed two technology sketches with the purpose of supporting presence-in-absence by employing graphical code languages: the Cube and the Picture Frame. The results of a subsequent evaluation involving potential users potentially inform the design of future presence-in-absence technologies.
\end{abstract}

Keywords Presence-in-absence · Intimate technology • Intimacy

\section{Introduction}

Presence-in-absence is a subjective feeling of a significant other when he or she is not physically co-present. This feeling is an important part of strong-tie social relationships [10]. We can be present-in-absence when we discuss work matters with colleagues over the telephone or when we write a letter to a close family member reflecting our feelings for them. In both cases, we sometimes experience the feeling of being present even though absent. Technologies can support such feelings

K. Garnæs $(\bowtie) \cdot$ O. Grünberger · J. Kjeldskov · M. B. Skov

Department of Computer Science, Aalborg University,

Fredrik Bajers Vej 7E, 9220 Aalborg East, Denmark

E-mail: kasper@garnaes.dk

E-mail: olgag@tiscali.dk

E-mail: jesper@cs.aau.dk

E-mail: dubois@cs.aau.dk

Tel.: + 45-9635-8080

Fax: + 45-9815-9889 in different ways and emerging technologies are increasingly being used to support intimate acts and presence-in-absence, for example mobile phones and the services SMS and MMS.

Research studies are currently showing a growing interest in investigating how to support intimacy and a class of technologies facilitating the feeling of presencein-absence has emerged. VIO [6] employs virtual minimal lights to suggest the presence of an intimate partner while The Love Eggs is a one-to-one voicemail system in tangible devices resembling eggs [7]. The idea is that communicating couples can send a message to his or her loved one by speaking into one egg causing the other egg to rotate. The partner can then listen to the recorded message by picking up the egg. The Hug-over-a-Distance proposes an inflatable jacket that emulates the feeling of a real hug [12]. The idea is that a partner over the Internet can trigger an event that causes the jacket to be inflated when the other partner reciprocates the hug. Finally, other studies try to replicate experiences relating to physical proximity such as blowing a kiss [3] or sleeping next to each other [4].

Despite such novel efforts, no established framework for designing presence-in-absence technologies exists. To investigate the design of technologies for presence-inabsence, we present in this paper two novel technologies designed to support the feeling of presence-in-absence through simple yet expressive and emotionally rich communication. First, we outline our understanding and use of presence-in-absence. Secondly, we illustrate the Cube and the Picture Frame, two paper prototypes for presence-in-absence, and finally we identify a number of lessons learned.

\section{Understanding presence-in-absence: contact, content, context}

Potentially, several dimensions describe and illustrate the inherent nature of presence-in-absence. Here we present an understanding of presence-in-absence 
integrating three separate but related themes namely contact, content, and context. We derived the three themes from collected material of the Strong-Tie Relationship project at University of Melbourne (see [5, 8, 12]). Vetere et al. [12] previously reported on the collection of ethnographic field data across six intimate couples over a 7-week period using cultural probes.

The primary data consisted of diaries, scrapbooks, photographs and multiple self-reports provided by the six intimate couples. Secondary data included interviews and focus groups conducted with all the couples. We analyzed the empirical data using a coding protocol constructed from previous work on intimacy and especially from work on social presence and connectedness $[1,9]$. Our coding and analysis process is further illustrated in [2]. On the basis of the coding and the analysis, we identified the three themes of presence-in-absence, contact, content, and context.

Contact defines the feeling of being in touch. Thus, intimate partners often feel the need to be in touch with their partner during the day despite separation due to work or leisure activities. The act of making a phone call or sending an email is a way for intimate partners to maintain contact throughout the day and thereby indicate "I am thinking of you". Reciprocated communication between intimate couples enhances the feeling of contact while e.g. unanswered phone calls or text messages have a negative emotional impact.

Content defines the form and substance of the communication between intimate couples. Owing to couples' intricate knowledge of one another, they are often able to communicate through code languages. Such code language enables the couple to express highly rich and affective content using seemingly simple expressions. Further, making a personal effort on the content, e.g. buying presents for one another, leaving special messages, or showing extra consideration when carrying out daily routines, has a positive effect.

Context defines factors in situations affecting intimate communication. Intimate partners have concerns about keeping their communication private, which are related to the context of the communication such as the physical surroundings as well as the technology employed. It is furthermore required that the intimate couples are in an available context-practical (reachable) as well as emotional (in a mood for communication) - in order to enable them to devote their attention to one another when communicating. Communication technologies should thus not be obtrusive, but let the partners decide when their context allows for communication with their intimate one.

\section{Designing technologies for presence-in-absence}

On the basis of the identified themes for presence-inabsence, we produced two different design ideas that would potentially support the special feeling of presence while absent. We used our findings from the analysis regarding intimate partners' use of private code languages as our starting point. We found that such codes are playful and inventive ways of coming up with simple expressions between partners containing great affective value. To distinguish our technology sketches or designs from well-established communication forms, such as textual or verbal communication, we based our use of code languages on graphical symbols. Other attempts for presence-in-absence technologies e.g. VIO [6] also employ a simple code language; however, our aim was to offer higher expressiveness than just a single bit communication.

Two diverging goals for the designs were in focus to explore trends in presence-in-absence technologies and symbol based code languages. The first design allowed complex and expressive communication while integrated into the users' virtual environment. The other design provided a simple communication form and should be a part of the users' physical environment. We named the two designs, respectively, the Cube and the Picture Frame.

In the following, we illustrate the two outlined designs. Both technology designs were illustrated, presented, and evaluated as paper prototypes. In this paper, we will not illustrate or discuss in detail how the design process of the paper prototypes took place; this paper serves to illustrate ideas for specific designs of presencein-absence technologies. Specific information on the design process and evaluation can be found in [2].

\section{The Cube}

The Cube aims to enhance the playful aspect of private code languages and intimacy in general [8] by combining symbol-based communication with rules and constraints similar to board games such as Scrabble. A brainstorming session facilitated the identification of a cube, which was selected due to the potential playfulness of three-dimensional spaces. Therefore, users of the Cube are encouraged to be creative when composing expressive messages to each other.

The Cube consists of a virtual three-dimensional cube shared between intimate couples. Each of the six sides of the cube contains nine squares and users can compose messages to each other by selecting and placing graphical symbols on these squares from a shared set of symbols. We included a number of predefined symbols for affective and communicative issues, e.g. a heart and numbers. Users can communicate with each other by placing an affective symbol on the cube and thereby saying that they are thinking of each other, but the squares also permit them to combine several symbols and thus create complex and expressive messages for each other (see Fig. 1). Users can even design new symbols to develop their own code language. The esoteric symbol-based communication also makes it difficult if not impossible to decode for outsiders and thus aims to ensure that the couples' communication stays private. 
The Cube is accessible through a broad range of technologies already employed in users' everyday life, such as laptops, mobile phones, PDAs. Thus, the Cube blends into the users' virtual environment and the possibility for being in contact is increased despite being physically separated. Users can also send notifications of messages left on the cube through other communication channels such as text messages or emails to promote reciprocal behavior.

Users have to access the Cube to both compose and read messages and thereby it is an action taken by the individual intimate whenever they can find the time for doing so in their everyday life. Thus, the system is meant to be unobtrusive. In the following, we present an envisioned use scenario to illustrate the potential use of the Cube for presence-in-absence.

\section{Envisioned use scenario}

Alice and Bob are planning to go on a vacation. They are really looking forward to getting some time off together and just this morning they talked about it over breakfast before heading to work. Bob is sitting in his office and has an idle moment and let his mind drift and the forthcoming vacation pops up in his thoughts. $\mathrm{He}$ decides to share his anticipation with Alice so he starts up the Cube, and logs into the system. Alice has left him a sweet message the day before and since he wants to save this he rotates the cube in order to find an unoccupied side. Bob composes the message to Alice by clicking on the squares on the side of the cube to open the symbol menu. After deciding which symbol to use, he clicks on it to place it on the square and moves on to the next square until he is satisfied with the message. Bob is really excited about their vacation and the message he just composed so he wants Alice to visit the Cube as soon as possible. Thus, he clicks the envelope in

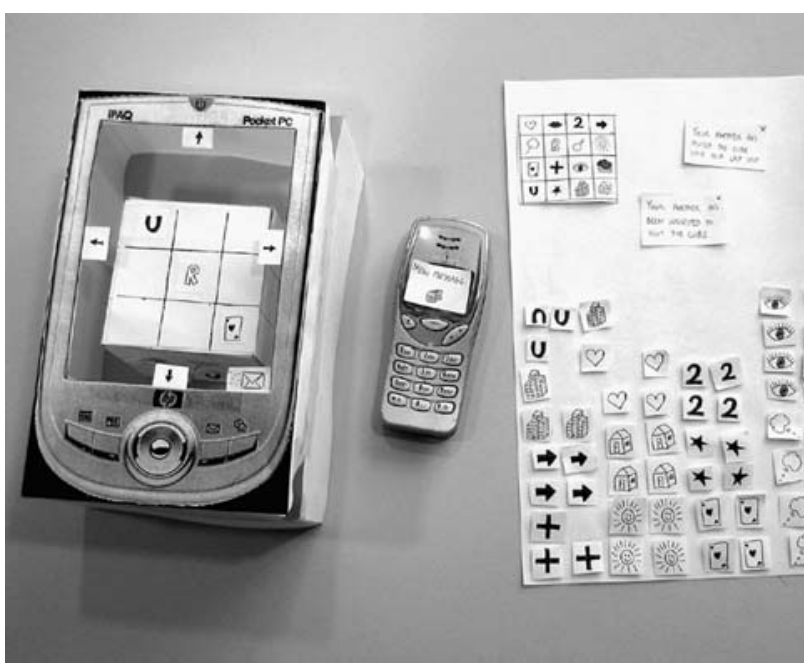

Fig. 1 Components of the Cube paper prototype the lower right corner of the interface knowing that a text message will be sent to Alice notifying her about his message. Bob continues his work with a smile and hopes Alice will soon react to his message.

\section{The Picture Frame}

We will now turn to the second design called the Picture Frame. This design is based on the observation that people often keep photographs of their loved ones at places where they are typically separated such as their offices. The Picture Frame aims to enhance the emotional value of photos by facilitating simple communication with the person depictured on the photograph while blending seamlessly into the physical environment and thus the users' other activities.

The Picture Frame is a networked digital touch screen with the appearance of a picture frame. The frame contains an image of the user's partner and through a simple user interface; the user is able to send singular graphical symbols to his or her partner's frame where they appear in a thought bubble beside the image of the user's head (Fig. 2). Thus, the Picture Frame provides a simple, quick, and easy way for intimate couples to communicate that they are thinking of each other using symbols based on their code language.

As the Picture Frame is intended to operate in offices and work places, it becomes a part of a partially public environment. However, as the communication between the user and his or her partner is conducted in their private code language, other people should not be able to fully understand the messages displayed on the Picture Frame. Activities at the workplace such as meetings or being out of the office may, however, render the user unavailable for communication and the Picture Frame takes this into account by letting users send their status to their partner's frame. When the user is occupied his or

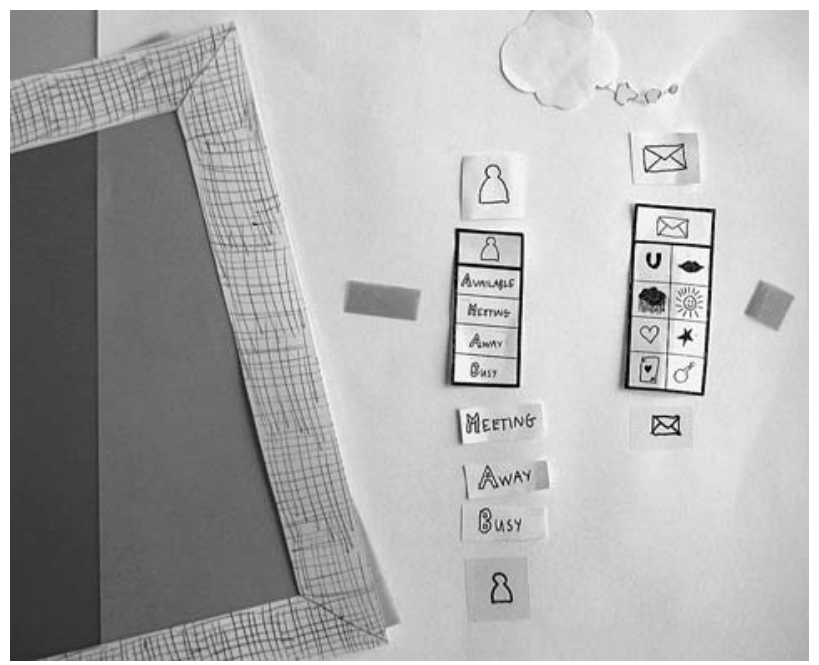

Fig. 2 Components of The Picture Frame paper prototype 
her partner's frame dims and the status is shown in a thought bubble. Furthermore, the frame does not emit any audible feedback when sending or receiving messages to avoid disturbing the user.

\section{Envisioned use scenario}

Alice and Bob work in separate parts of the city. Alice sits in her office engaged in a phone conference when her eyes catches the Picture Frame placed on her desk in front of her with a really goofy picture of Bob in it. Just as she lets her eye wander off a thought bubble appears in the frame from Bob with a star in it. Alice smiles and guesses that the comments she made for Bobs presentation yesterday turned out to be bang on. While she is still engaged in the phone conference she pressed the envelope in the lower right corner of the picture frame. As a response to this a symbol menu pops up and Alice presses the heart and then the envelope in the top of the menu. As the menu disappears from the touch screen she smiles knowing that a heart will pop on at the picture of her in Bob's office. After the phone conference is over Alice heads out to lunch. Before leaving the office she presses the person icon in the lower left corner of the frame. When the menu pops up she presses the away button and the person icon again. Subsequent she leaves the office knowing that Bob will know that she is out due to the fading of the picture of her in his frame.

\section{Evaluating the paper prototypes}

We conducted a number of evaluations of the two designs to explore their potential as presence-in-absence technologies. In order to achieve qualitative feedback of the ideas behind each of the prototypes, we had potential users interact with the paper prototypes through the course of a usability test as proposed by Snyder [11]. We recruited four participants among researchers at the Department of Information Systems, University of Melbourne. All four participants evaluated both design ideas counter-balanced having two participants interact with the Cube and then with the Picture Frame and the other participants vice versa.

During these tests, the participants were instructed to interact with the paper prototypes in a range of small scenarios (Figs. 3 and 4). These scenarios consisted of imaginary communication situations based on the envisioned use scenarios previously described. During and after the test, participants were enquired and interviewed about their opinions of the two designs. The participants were all involved in intimate relationships and thus potentially in possession of in-depth knowledge about the intricate nature of intimate relationships.

Our results indicated that the participants believed that both prototypes had the potential to support presence-in-absence. However, this was expressed in different ways. Overall, the participants were positive about

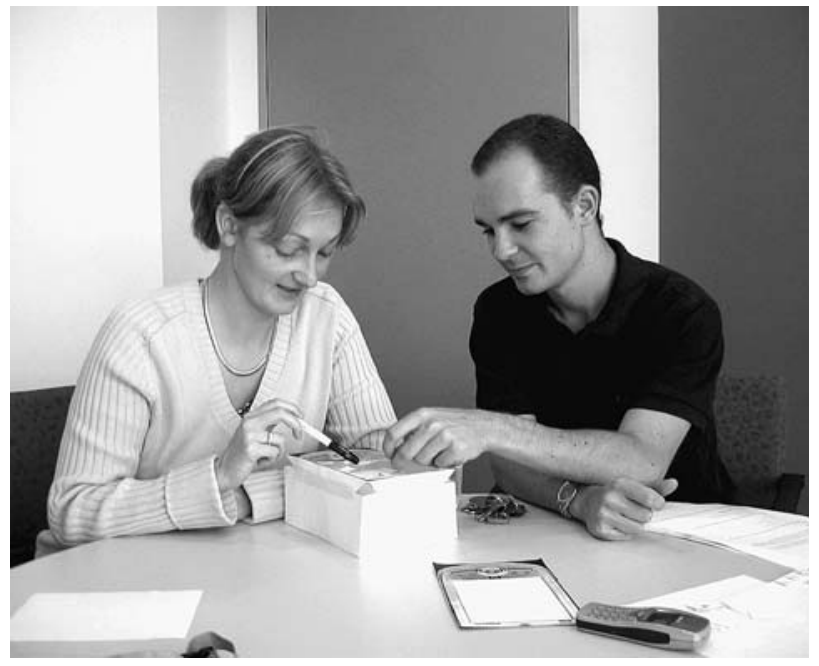

Fig. 3 Evaluating the Cube

the design idea of the Cube as the expressiveness and long-term possibilities of the design idea allowed intimate partners to enhance their code languages. The participants thought that the Cube provided a sense of playing together and that this worked as a good way to be in contact with each other. The participants furthermore believed that the Cube had the potential to give them a sense of their partner when not together. This is consistent with the envisioned use scenario where Bob wishes to give Alice a sense of his anticipation of their forthcoming vacation.

In regard to design idea of the Picture Frame, the participants liked the visualization of their partner, the ease of sending messages to stay in touch, and the direct connection facilitated by the design idea. The participants articulated that the Picture Frame offered a good way to be connected and that it facilitated the possibility for the intimates to express their state of mind - although in a very simple way. Communicating

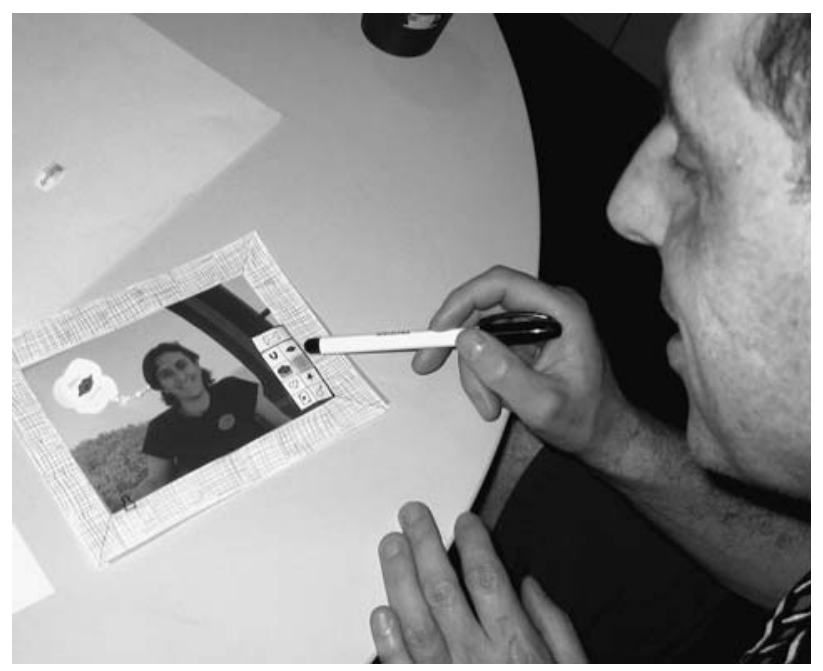

Fig. 4 Evaluating The Picture Frame 
by using the Picture Frame was compared to giving a little blink in the partners' life saying: "Yeah, I'm here", as depictured by the envisioned use scenario of the Picture Frame.

\section{Lessons learned}

On the basis of our paper prototype designs and the evaluations, we have identified three major lessons in regard to presence-in-absence technologies. These relate (1) symbol-based code languages have potentials, (2) mobile devices dedicated to communication between intimate partners are preferred, and (3) there is a conflict between the themes of contact and content.

First, the participants were very positive towards communication through symbol based code languages. They found that pictures and symbols were more expressive than words and they liked the creative possibilities they offered. The positive response is interesting in the design of presence-in-absence technologies as they address issues of all three themes of contact, content and context. Participants found that they required little effort to use, conveyed rich meanings to the users, and ensured privacy. Thus, the possibilities of symbol-based code languages presented in the envisioned use scenarios of the two designs turned out to be very promising. Consequently, we considered symbol-based code languages as an interesting alternative to the textual and verbal communication forms used in most contemporary well-established technologies, such as mobile phones and text messages.

Secondly, the participants expressed that the Picture Frame facilitated a direct connection between intimate partners and they liked that they had a specific device for communicating with their partner. However, one consistent problem of the Picture Frame was that the device is stationary and all the participants mentioned that they themselves or their partner was usually at different locations during the day, which renders regular use problematic. In regard to the envisioned use scenario of the Picture Frame it is also relevant for Alice to be able to communicate with Bob when she e.g. is out for lunch. In regard to this the possibility for accessing the Cube through different devices was positively met by the participants. The problem with the Cube was that the devices used to access the system were not used solely for communication between intimate partners. This suggests that the affective value Bob experiences when using the Cube in the envisioned use scenario would be increased if he accessed the Cube from a device used solely to communicate with Alice. Consequently, a system with the purpose of supporting presence-in-absence should be a portable device dedicated to communication between intimate partners.

Thirdly, we identified an interesting fundamental conflict in the design of presence-in-absence technologies in relation to the two themes contact and content. The designs relate to the themes in very different ways and this was reflected in the participants' articulations. The Cube facilitates the communication of expressive and playful content with possibilities for future enhancement but this also makes it mentally demanding and perhaps even obtrusive to communicate. Thus, the Cube would not be able to provide a sense of contact between intimate partners. In the envisioned use scenario of the Cube, Bob composed his message to Alice by putting several symbols together to create a specific meaning, but it is questionable whether this process is too complex to perform continually throughout the day. Conversely, the Picture Frame offers a quick and easy way of communicating which provides a sense of contact but the content could be too simplistic on a long-term basis. Alice and Bob each used a singular symbol to communicate to each other in the envisioned use scenario of the Picture Frame, but the evaluation suggested that the value of sending e.g. a singular heart looses value after a period of time, and it is difficult to enhance and further develop a code language based on singular symbols. On the basis of our evaluation, we are unable to clarify whether the two themes are contradictory in nature or if designs can be produced which successfully supports both themes of presence-in-absence.

\section{Conclusion}

Presence-in-absence is a subjective feeling of a significant other when he or she is not physically co-present and this feeling is an important part of strong-tie social relationships. Communication and information technologies can support such feelings in different ways and emerging technologies are increasingly being used to support intimate acts and presence-in-absence, for example mobile phones and the services SMS and MMS. However, no established framework for designing such presence-in-absence technologies exists.

Therefore, to investigate the design of technologies for presence-in-absence, we have presented two technologies designed to support the feeling of presence-in-absence through simple yet expressive and emotionally rich communication - we named these the Cube and the Picture Frame. The two designs were based on an analysis of rich data describing how intimate partners interact throughout their everyday life. From a number of evaluations, we learned three important lessons throughout our work of this article in regard to requirements for presence-in-absence systems. First, symbol-based code languages address needs concerning contact, content and context. Second, a device for supporting presence-inabsence should be both mobile and dedicated to communication between intimate partners. Third and finally, our evaluation pointed at a conflict between the themes contact and content due to contradictions between simply staying in contact and conveying rich content.

Our research is characterized by a number of limitations. First, it is difficult to explore and investigate the actual potentials of presence-in-absence technologies 
without full implementation of the design ideas. Our evaluation can be considered a tentative evaluation with the purpose of identifying immediate potentials for designing such technologies, but fully functional technologies should be implemented and evaluated to better understand presence-in-absence technologies. Secondly, the inherent nature of presence-in-absence and intimacy probably requires longitudinal studies of the adaptation of such technologies. It is straightforward to assume that the use and adaptation of such technologies would change over time as they become a natural part of your environment. A snapshot evaluation like the one we conducted in our experiment can only provide very limited insight into such considerations. Thus, such challenges and limitations could potentially inform further studies of the design of presence-in-absence technologies.

\section{References}

1. Biocca F, Burgoon J, Hrms C, Stoner M (2001) Criteria and scope conditions for a theory and measure of social presence. In: Proceedings of PRESENCE 2001, $4^{\text {th }}$ international workshop

2. Garnæs KN, Grünberger O (2005) Designing for intimacy and presence in absence. Department of Computer Science, Aalborg University
3. Gaver B (2002) Provocative awareness. Comput Support Coop Work 11:475-493

4. Goodman E, Misilim M (2003) The sensing bed. In: Proceedings of UbiComp 2003 workshop

5. Howard S, Kjeldskov J, Skov MB, Garnæs KN, Grünberger O (2006) Negotiating presence-in-absence: contact, content and context. In: Jeffries R (ed) In: Proceedings of the human factors in computing systems (CHI'06), ACM Press, Montreal, pp 909-912 (CHI note)

6. Kaye J, Levitt MK, Nevins J, Golden J, Schmidt V (2005) Communicating intimacy one bit at a time. CHI '05 extended abstracts on human factors in computing systems, pp 15291532

7. Kaye J, Goulding L (2004) Intimate objects. In: Proceedings of the 2004 conference on designing interactive systems, pp 341344

8. Kjeldskov J, Vetere F, Gibbs M, Howard S, Pedell S, Mecoles $\mathrm{K}$, Bunyan M (2004) Mediating intimacy: designing technologies to support strong tie relations, OZCHI

9. Moss BF, Schwebel AI (1993) Defining intimacy in romantic relationships. Fam Relat 42:31-37

10. Register LM, Henley TB (1992) The phenomenology of intimacy. J Soc Pers Relat 9:467-481

11. Snyder CR (2003) Paper prototyping: fast and simple techniques for designing and refining the user interface. Morgan Kaufmann, San Francisco

12. Vetere F, Gibbs MR, Kjeldskov J, Howard S, Mueller F, Pedell S, Mecoles K, Bunyan M (2005) Mediating intimacy: designing technologies to support strong-tie relationships. In: Proceedings of the SIGCHI conference on human factors in computing systems, pp 471-480 